\title{
Loss of TACSTD2 contributed to squamous cell carcinoma progression through attenuating TAp63-dependent apoptosis
}

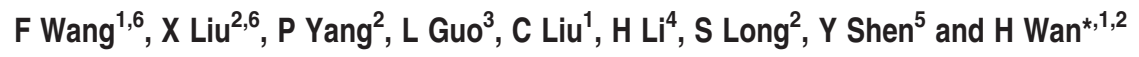

Tumor-associated calcium signal transducer 2 (TACSTD2), a calcium signal transducer, is universally expressed in stratified squamous epithelia of many organs, including skin, esophagus and cervix. Although TACSTD2, was reported to be overexpressed in many epithelial tumors, which has increased interest in using it as a molecular target for cancer therapy, the role of TACSTD2 in carcinogenesis of squamous cell carcinoma (SCC) is largely unclear and controversial. To explore the role of TACSTD2, temporal-spatial expression of TACSTD2 was analyzed in both normal and SCC tissues. Our data demonstrate that Tacstd2 expression and membrane localization are tightly associated with stratified epithelial homeostasis, while loss of TACSTD2 was identified in poorly differentiated SCC tissues collected from cervix, esophagus, head and neck. Gradual loss of TACSTD2 was correlated with stepwise progression of SCC. Consistent with these in vivo observations, our data show that inhibition of Tacstd2 expression significantly inhibited chemotherapeutic reagent-induced apoptosis, and TACSTD2 regulated apoptotic gene expression through P63 containing the transactivation domain (TAp63). These findings indicated that loss of TACSTD2 could promote SCC progression and treatment resistance through attenuating chemotherapeutic reagent-induced apoptosis through TAp63, and TACSTD2 could be used as a marker for pathological grading of SCC.

Cell Death and Disease (2014) 5, e1133; doi:10.1038/cddis.2014.96; published online 20 March 2014

Subject Category: Cancer

Squamous cell carcinoma (SCC) is derived from squamous epithelia in many essential organs, including skin, esophagus, lung, mouth and cervix. It is the most common metastasizing cancer worldwide, and its survival rate has not improved significantly in the past 30 years. ${ }^{1,2}$ Although SCC arises from different organs, SCCs share many phenotypic and molecular characteristics with each other. ${ }^{3}$ Further understanding of the common molecular mechanism associated with squamous epithelial homeostasis and progression of SCC will be of great benefit for better prevention and treatment of SCC.

Tumor-associated calcium signal transducer 2 (TACSTD2) is generally expressed in normal squamous epithelia of many organs, including skin, pharynx, esophagus, vagina and so on, indicating that TACSTD2 may have important roles in squamous epithelial homeostasis. In support of this hypothesis, studies demonstrated that TACSTD2 is required for maintenance of the epithelial barrier through regulation of proper localization of tight junction proteins, whereas loss of TACSTD2 resulted in susceptibilities to several epithelial diseases, including epithelial tumor and corneal dystrophy. ${ }^{4-6}$ Contradictory evidence exists, however, showing increased expression of Tacstd2 in several epithelial tumors, increasing the interest in using it as a molecular target for cancer therapy. ${ }^{7,8}$ Therefore, the roles of TACSTD2 in normal biology and neoplastic progression remain unclear.

Keratinocyte differentiation and apoptosis are required for normal tissue homeostasis. Abnormal regulation of these biological events is associated with progression of SCC. In this study, the in-depth physiopathological analysis of SCC progression and exploration of the associated molecular mechanism were carried out to explore the function of TACSTD2 in SCC. Our study demonstrated that temporal-spatial expression of TACSTD2 was associated with stratified epithelial homeostasis. Progressive loss of TACSTD2 was correlated with SCC progression. Inhibition of Tacstd2 expression significantly inhibited chemotherapeutic reagent-induced keratinocyte apoptosis and TACSTD2 regulated apoptotic gene expression through P63 containing the

\footnotetext{
${ }^{1}$ Key Laboratory of Obstetric, Gynecologic and Pediatric Diseases and Birth Defects of the Ministry of Education, West China Second University Hospital, Sichuan University, Chengdu, Sichuan 610041, China; ${ }^{2}$ Department of Obstetrics and Gynecology, West China Second University Hospital, Sichuan University, Chengdu, Sichuan 610041, China; ${ }^{3}$ Department of Pathology, The First Affiliated Hospital of SooChow University, Suzhou 215006, China; ${ }^{4}$ Department of Anesthesiology, West China Second University Hospital, Sichuan University, Chengdu, Sichuan 610041, China and ${ }^{5}$ Department of Pathology, West China Second University Hospital, Sichuan University, Chengdu, Sichuan 610041, China

${ }^{*}$ Corresponding author: $\mathrm{H}$ Wan, Key Laboratory of Obstetric, Gynecologic and Pediatric Diseases and Birth Defects of the Ministry of Education, West China Second University Hospital, Sichuan University, No.17, Section 3, South Renming Road, Chengdu, Sichuan 610041, China. Tel: +86 2885503629 ; Fax: +86 28 85501863; E-mail: huajingwan@scu.edu.cn

${ }^{6}$ These authors contributed equally to this study.

Keywords: TACSTD2; squamous cell carcinoma; apoptosis; TAp63; tumor progression

Abbreviations: SCC, squamous cell carcinoma; CIN, carcinoma in situ; TACSTD2, tumor-associated calcium signal transducer 2; TAp63, P63 containing the transactivation domain; KRT10, cytokeratin 10; SFN, stratiffin; IVL, involucrin; KRT5, cytokeratin 5; DSC2, desmocollin 2; BAX, BCL2-associated X protein; CD95, cluster of differentiation 95; KI67, antigen identified by monoclonal antibody Ki-67

Received 19.1.14; accepted 29.1.14; Edited by M Agostini
} 
transactivation domain (TAp63), indicating that TACSTD2 has important roles in SCC progression and treatment resistance through regulating TAp63-dependent apoptosis, and TACSTD2 could be used as a marker for pathological grading of SCC.

\section{Results}

Loss of TACSTD2 was identified in SCCs collected from different organs. In order to determine whether disregulation of TACSTD2 is associated with SCC, immunohistochemistry staining of TACSTD2 was performed on sections of poorly differentiated SCCs collected from cervix $(n=20)$, esophagus $(n=5)$ and head-and-neck $(n=6)$. Normal epithelial tissues adjacent to SCCs were used as internal controls. In normal stratified epithelium, strong TACSTD2 staining was identified on the cell membrane of differentiated epithelial cells (Figures 1a, c and e, arrowhead). In poorly differentiated SCCs, TACSTD2 staining was absent in most of the cancer cells (Figures $1 b, d$ and $f$ ). Weak cell membrane or cytoplasmic staining was only detected in a few scattered epithelial cells (Figures $1 b$, $d$ and $f$, arrowhead). These data indicated that loss of TACSTD2 could be a common phenomenon of poorly differentiated SCC at different anatomical sites.

Loss of TACSTD2 is associated with SCC progression. Pre-cancerous lesions at early stages of SCC development were easily collected from cervical region. In order to determine whether expression of TACSTD2 is significantly associated with stepwise progression SCC, well-staged cervical tissues were collected. Immunohistochemistry staining of TACSTD2 was analyzed in controls, carcinoma in situ (CIN) and SCC tissues collected from the cervix. Compared with CONs (Figure 2a), epithelial cells with cell membrane staining of TACSTD2 were gradually decreased from CIN1 to SCC (Figures $2 b-e$ ). In order to quantify the loss of TACSTD2-positive cells, average TACSTD2-negative epithelial cell ratios for CON $(n=23)$, CIN1 $(n=9)$ and CIN2/3 $(n=27)$, and SCC $(n=20)$ were calculated as described in Materials and Methods section, respectively. Significant differences among the groups were found by the ANOVA test $(F=114.98, P<0.05)$, and significant differences between each of the groups were identified by the Student-Newman-Kabul's multiple comparison test $(P<0.05$; Figure 2f), indicating loss of TACSTD2 was
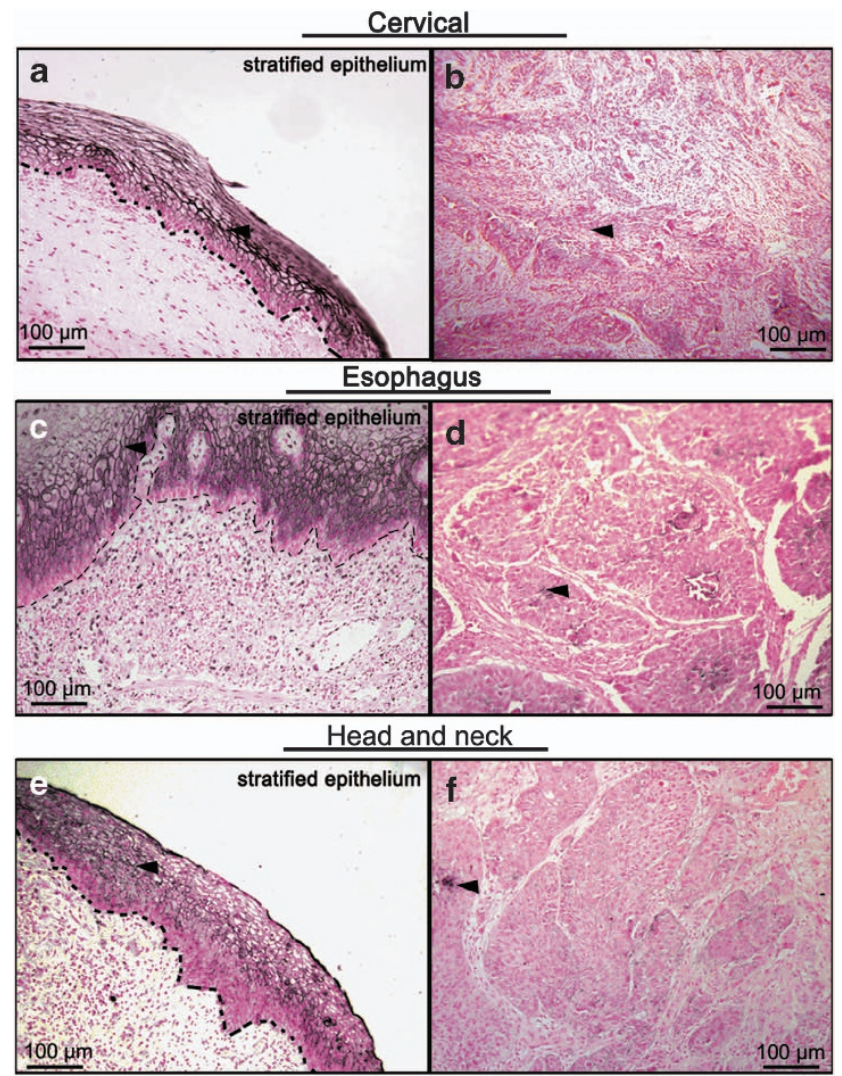

Figure 1 Immunohistochemistry of TACSTD2 on tissues of SCC. Paraffin sections of poorly differentiated SCCs from cervix $(n=20)$, esophagus $(n=5)$ and head-and-neck $(n=6)$ were stained with TACSTD2 antibody. Representative figures for adjacent normal stratified epithelium (a, $\mathbf{c}$ and $\mathbf{e})$ and poorly differentiated SCCs (b, $\mathbf{d}$ and $\mathbf{f})$ are shown. The basement membranes of normal stratified epithelium are highlighted with dotted lines. Epithelial cell with staining of TACSTD2 is indicated with arrowheads

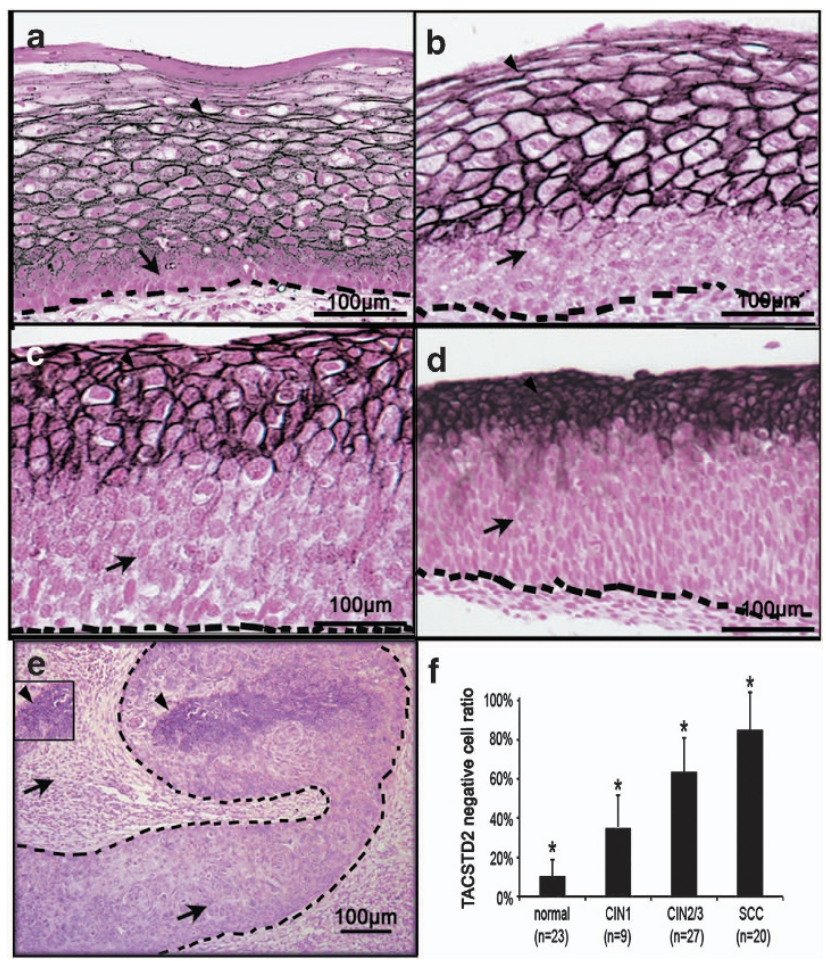

Figure 2 Loss of TACSTD2 staining associated with cervical SCC progression. Paraffin sections from cervical precancerous and cancerous lesions were prepared and immune-stained with TACSTD2 antibody. Representative figures for CON (a), CIN1 (b), CIN2 (c), CIN3 (d) and SCC (e) are shown. A bar graph showing the TACSTD2-negative cell ratios of CON $(n=23), \operatorname{CIN} 1(n=9), \operatorname{CIN} 2 / 3(n=27)$ and SCC $(n=20)$ are presented as the mean \pm S.E.M. (f) Significant difference was identified $\left({ }^{*} P<0.05\right)$. Epithelial cells with membrane staining of TACSTD2 are indicated with arrowheads and epithelial cells without membrane staining of TACSTD2 are indicated with arrows 
positively correlated with progression of SCC. Interestingly, although loss of TACSTD2 staining was increased significantly as cervical cancer progressed, the intensity of staining in the remaining TACSTD2-positive cells appeared to be increased from CON to CIN3 (Figures $2 a-d$, arrowhead), indicating mechanistically that induction of Tacstd2 expression in response to tissue stress remains intact in normal keratinocytes within the lesions.

Heterogeneity of TACSTD2 staining was observed in many SCCs, representing a mixture of biologically different cell populations. In order to determine whether there are differences in TACSTD2 staining between poorly differentiated SCCs and moderately differentiated SCCs, average TACSTD2-negative epithelial cell ratios were compared. The TACSTD2-negative epithelial cell ratio was significantly lower in moderately differentiated SCCs $(n=5)$ compared with that of poorly differentiated SCCs ( $n=15$; Figure 3$)$.
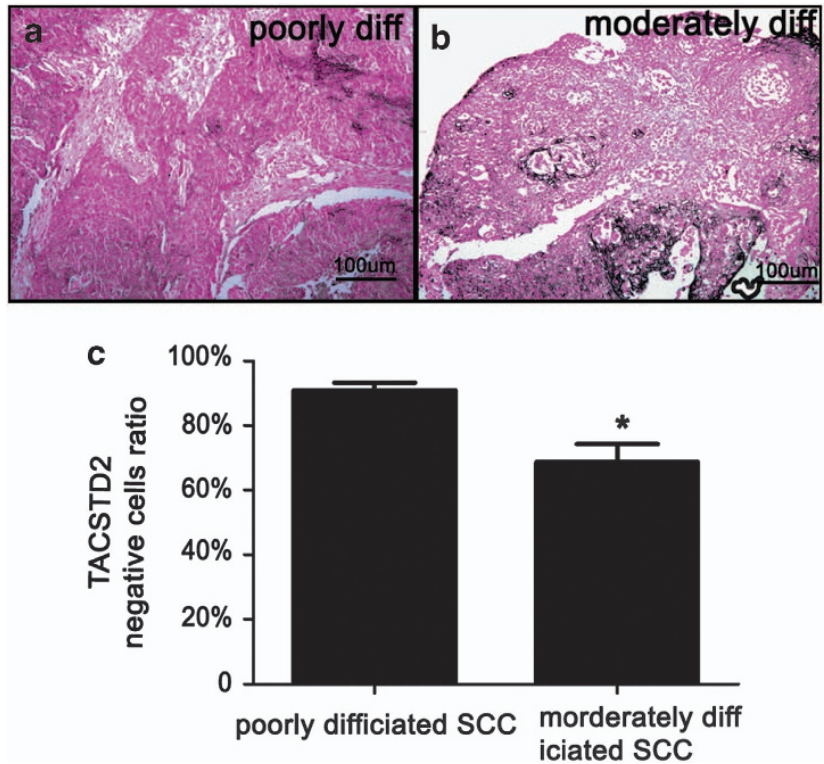

Figure 3 Difference of TACSTD2 staining between poorly differentiated and moderately differentiated SCCs. Paraffin sections of cervical SCCs were immunostained with TACSTD2 antibody. Representative figures for poorly differentiated (a) and moderately differentiated (b) SCCs are shown. A bar graph showing the TACSTD2-negative cell ratios of poorly differentiated $(n=15)$ and moderately differentiated $(n=5)$ SCCs are presented as the mean \pm S.E.M. (c) Significant differences were identified $\left({ }^{*} P<0.05\right)$
These data demonstrated that gradual loss of TACSTD2positive epithelial cells was associated with SCC progression, indicating that loss of TACSTD2 may have a critical role in SCC progression and suggesting that TACSTD2 could be used as a marker for pathological grading of SCC.

Expression of TACSTD2 is associated with epithelial homeostasis. Most of the SCCs occur around squamocolumnar junctions at different anatomic places including anus, cervix and esophagus. ${ }^{9}$ In order to understand the roles of TACSTD2, expression of TACSTD2 was analyzed using tissues, which contain the squamocolumnar junction $(n=6)$. In stratified squamous epithelia, cell membrane staining of TACSTD2 was detected in differentiated keratinocytes in the stratum spinosum, stratum granulosum and stratum corneum (Figure 4a, arrowhead), whereas staining of TACSTD2 was absent in less differentiated keratinocytes in the stratum basal/parabasal layers (Figure 4a, arrow). TACSTD2 staining was absent in cells undergoing squamous metaplasia at the squamous-columnar junction (Figure 4b, arrow). These results indicated that expression and cell membrane localization of TACSTD2 is tightly associated with differentiated keratinocytes. In columnar epithelium, staining of TACSTD2 was detected on the surface, directly facing the cervical canal (Figures $4 \mathrm{~b}$ and $\mathrm{c}$, arrowhead). On the other hand, no TACSTD2 staining was detected in the columnar epithelium invaginating into the substance of cervical stroma (Figure 4c, arrow).

In conclusion, expression of TACSTD2 was tightly associated with keratinocyte differentiation, and it was expressed in surface epithelium directly facing the external environment. This spatial tissue localization of TACSTD2 indicates that significantly TACSTD2 may have potential roles in epithelial homeostasis. This concept was supported by our findings that significantly increased accumulation of TACSTD2-positive keratinocytes were observed 4 days after less invasive epidermal injury by tape stripping (Supplementary Figure 2).

Inhibition of TACSTD2 did not affect keratinocyte differentiation. Loss of TACSTD2 was associated with SCCs at different anatomic sites. Consistent with in vivo observations, strong cell membrane staining of TACSTD2 was detected in HaCaT cells (Figure 5a), a keratinocyte cell line that highly preserved differentiation capability, ${ }^{10,11}$ but significantly weakened or lost in Siha cells, a squamous cancer cell line (Figure 5b, arrow). In order determine whether TACSTD2 is required for keratinocyte differentiation,

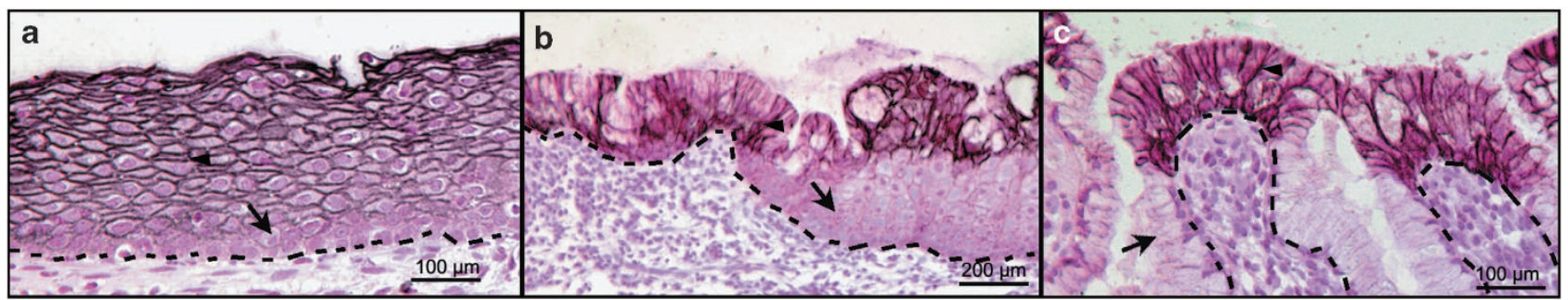

Figure 4 Immunohistochemistry of TACSTD2 on tissues with squamocolumnar junction. Paraffin sections of tissues containing squamocolumnar junctions were stained with TACSTD2 antibody. Representative figures for cervical stratified squamous epithelium (a), metaplastic area (b) and columnar epithelium (c) are shown. The basement membranes are highlighted with dotted lines. The arrow indicates negatively stained epithelial cells and the arrowhead indicates positively stained epithelial cells 
siRNAs to silence Tacstd2 expression was used to transfect $\mathrm{HaCaT}$ cells cultured in medium containing different calcium concentration, and the expression of genes associated with keratinocyte differentiation was analyzed. RT-PCR analysis demonstrated that expression levels of cytokeratin 10 $(K r t 10)$, stratiffin (Sfn) and involucrin (IVI), key markers of differentiated keratinocytes, ${ }^{12-14}$ were induced by calcium, but were not affected by Tacstd 2 silencing (Figures $5 \mathrm{~g}-\mathrm{i}$ ). Although expression levels of cytokeratin 5 (Krt5) and desmocollin 2 (Dsc2), markers for undifferentiated basal layer epithelial cells, ${ }^{13,15}$ were slightly inhibited by calcium, and were increased significantly after Tacstd2 silencing.

P63 is a transcription factor required for keratinocyte differentiation. ${ }^{16}$ RT-PCR analysis demonstrated that $\triangle N p 63$, the isoform that is critical for keratinocyte differentiation, ${ }^{17}$ was inhibited by calcium and was not affected by Tacstd2 silencing, but TAp63, an isoform of P63 that functions in regulation of keratinocyte apoptosis, ${ }^{18}$ was significantly induced by calcium and was significantly decreased after Tacstd2 silencing (Figure 5k), indicating that TACSTD2 may have roles in keratinocyte apoptosis. These data demonstrated that inhibition of Tacstd2 did not affect keratinocyte differentiation, but induced expression of genes required to maintain the keratinocyte in an undifferentiated status.

Loss of TACSTD2 inhibited chemotherapeutic reagentinduced keratinocyte apoptosis. Proteins involved in apoptosis were enriched in the stratum spinosum and stratum granulosum, ${ }^{19}$ where TACSTD2 was found to be highly expressed. In order to test the roles of TACSTD2 in apoptosis, expression of genes critical for apoptosis were analyzed after Tacstd2 silencing in $\mathrm{HaCaT}$ cells. The number of cleaved caspase-3-positive cells, as assessed by immunofluorescent staining, was significantly decreased (Figures $6 \mathrm{a}$ and b). Similarly, mRNA levels of BCL2-associated $X$ protein (Bax) and cluster of differentiation 95 (Cd95), two important genes involved in pro-apoptosis signaling, ${ }^{20,21}$ were decreased significantly (Figure 6c). In contrast, no changes in antigen identified by monoclonal antibody Ki-67 (KI67) and CYCLIND1, two proliferation markers, ${ }^{22,23}$ were observed by western blot analysis (Figure 6d). a

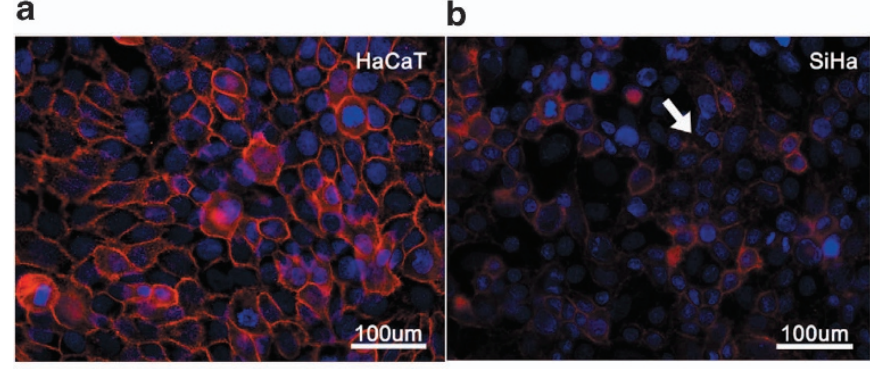

c

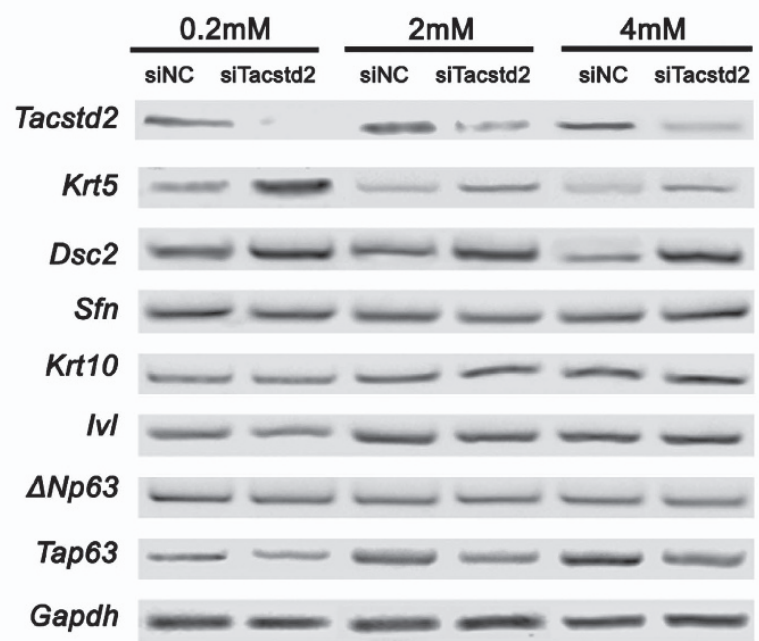

d
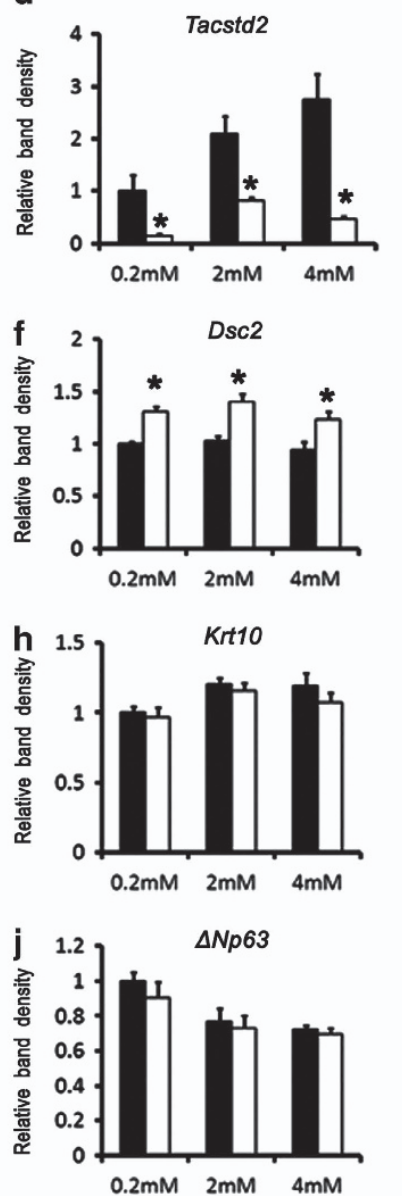
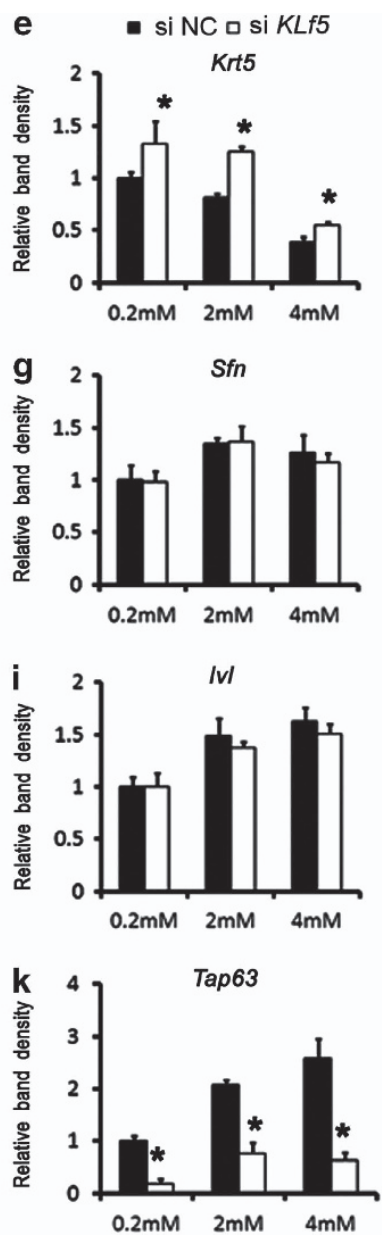

Figure 5 Effects of TACSTD2 on keratinocyte differentiation. (a and $\mathbf{b}$ ) Immunofluorescent staining of TACSTD2 (red) and nuclear counterstaining of DAPI (blue) on $\mathrm{HaCaT}$ and Siha cells. Arrow indicated Siha cell membrane lack of cytomembrane staining of TACSTD2. (c-k) RT-PCR was used to quantitate Krt5, Dsc2, Krt10, Sfn, Ivl, TAp63, $\triangle$ Np63 and Gapdh mRNAs in control and Tacstd2-silenced HaCaT cells cultured in mediums with different calcium concentrations. Relative mRNA levels are shown as mean \pm S.E.M. compared by a two-tailed Student's $t$-test $\left({ }^{*} P<0.05, N=3\right)$. Two Tacstd2 siRNA oligos as described in Materials and methods section were tested, and similar phenotypes were observed. Higher efficiency of Tacstd2 knockdown was observed using Tacstd2 siRNA oligo (ID HSS106222), and a representative illustration is shown 

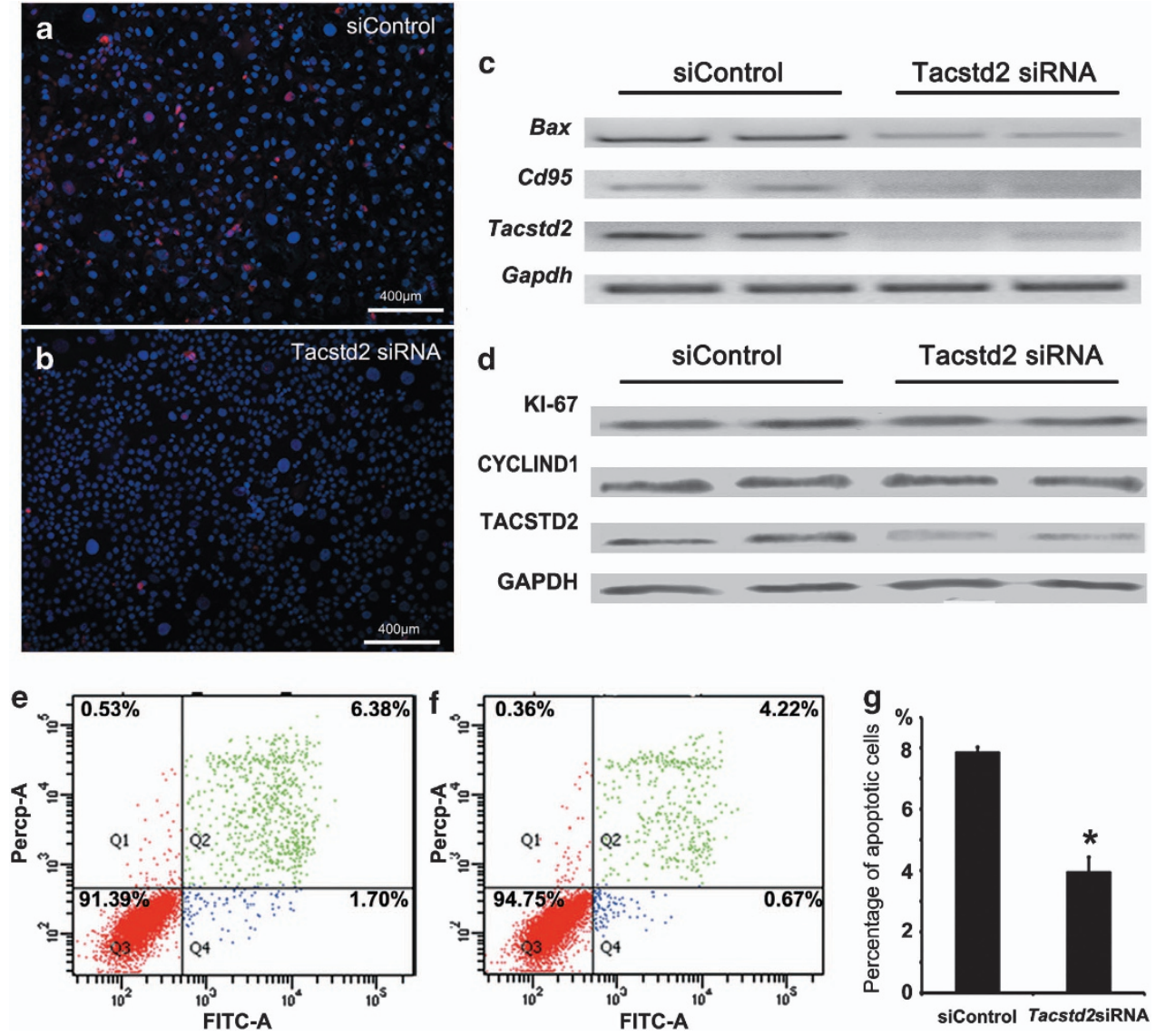

Figure 6 Effects of TACSTD2 on apoptosis and proliferation. (a and b) Immunofluorescent staining of cleaved caspase-3 (red) in control and Tacstd2-silenced HaCaT cells. Nuclei were counterstained with DAPI (blue). Two Tacstd2 siRNA oligos were tested as described in Materials and Methods section, and similar phenotype were observed. Higher efficiency of Tacstd2 knockdown was observed by using Tacstd2 siRNA oligo (ID HSS106222). (c) RT-PCR analysis of Bax, Cd95 and Gapdh mRNAs in control and Tacstd2-silenced HaCaT cells. (d) Western blot analysis of KI67, CYCLIND1, TACSTD2 and GAPDH in control and Tacstd2-silenced HaCaT cells. (e and f) Representative figures for gemcitabine-induced apoptosis, analyzed by flow cytometry using Annexin V-FITC/PI, are shown for control and Tacstd2-silenced HaCaT cells. (Q1: necrosis; Q2: late apoptosis; Q3: healthy cells; Q4: early apoptosis). (g) A bar graph showing percentage of gemcitabine-induced apoptosis in control and Tacstd2-silenced HaCaT cells. A significant difference was identified between control and Tacstd2-silenced HaCaT cells by using the Student's $t$-test $\left({ }^{\star} P<0.05, N=3\right)$

Gemcitabine is a cytotoxic medicine used to induce apoptosis in chemotherapy of SCCs. ${ }^{24,25}$ In order to test if TACSTD2 is functionally required for cytotoxic reagentinduced apoptosis, the effect of Tacstd2 silencing on gemcitabine-induced apoptosis was assessed by flow cytometry. Compared with controls, gemcitabine-induced apoptosis were significantly decreased in Tacstd2-silenced (Figures $6 \mathrm{e}-\mathrm{g}$ ) cells, indicating TACSTD2 regulated chemotherapeutic reagent-induced keratinocyte apoptosis, and may have critical roles in treatment resistance of SCCs.

TACSTD2 affects genes expression partially through regulating TAP63. Previous studies showed that TAp63 was a crucial regulator of squamous epithelium homeostasis. ${ }^{18,26}$ In order to test if TACSTD2 functions through TAp63, a TAp63-expressing plasmid was transfected into $\mathrm{HaCaT}$ cells after Tacstd2 silencing, and cleaved caspase-3, Bax, Cd95, Dsc2 and Krt5 were analyzed by immunofluorescent staining or RT-PCR. TACSTD2-dependent activation of caspase-3 was partially rescued by forced expression of TAp63 (Figures 7a-c). TACSTD2-dependent expression of Bax, Cd95 and Dsc2 were partially rescued by forced expression of TAp63 (Figures 7e and f), but TACSTD2regulated $\mathrm{Krt5}$ expression is independent of TAp63. These observations indicate that TACSTD2 regulates keratinocyte gene expression partially through TAP63.

\section{Discussion}

Understanding the molecular mechanism is required for better diagnosis and treatment of SCC. Animal model studies demonstrated that loss of TACSTD2 did not alter the incidence and natural history of papilloma formation, but enhance malignant phenotype of skin cancers through promoting EMT. ${ }^{6}$ Consistent with these, this study demonstrated that loss of TACSTD2 is a hallmark for the progression of human SCC, and it has critical roles in SCC progression and treatment resistance by attenuating chemotherapeutic reagent-induced keratinocyte apoptosis through TAp63.

\section{Gradual loss of TACSTD2 is a hallmark of the stepwise} progression of SCC. Carcinogenesis of SCC is a multistage process. Gradual accumulation of tumor cells caused by abnormalities in the progenitor cells of the basal layer is 

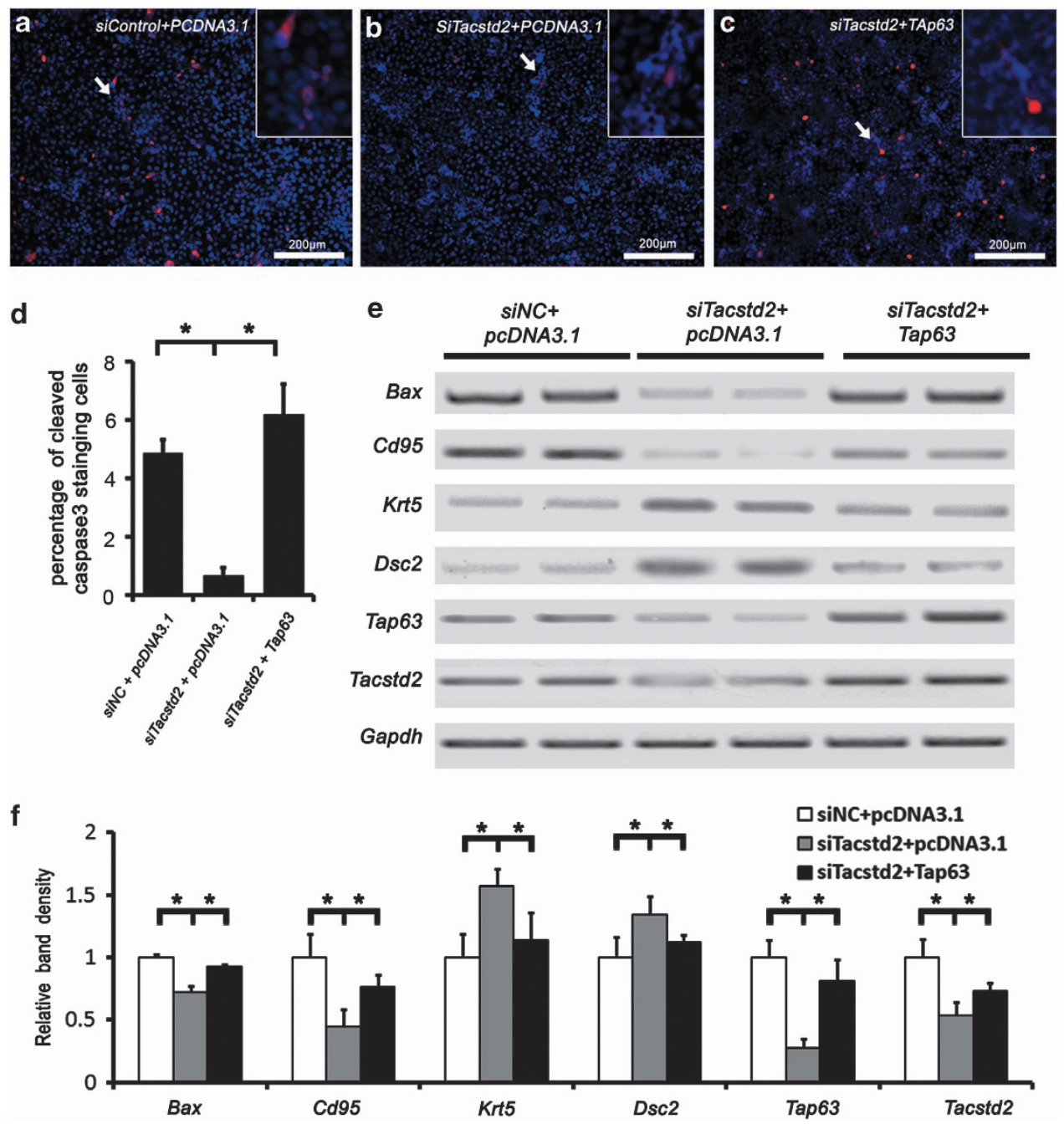

Figure 7 Induced expression of TAp63 in HaCaT cells rescued TACSTD2-dependent caspase-3 activation and apoptotic gene expression. (a-c) Immunofluorescent staining of cleaved caspase-3 (red). Nuclei were counterstained with DAPI (blue). Inserts are higher magnifications of the cleaved caspase-3-positive cells. (d) A bar graph showing percentage of cleaved caspase-3-positive cells. Significant differences were identified between control and Tacstd2-silenced HaCaT cells, between Tacstd2-silenced HaCaT cells and TAp63 rescued cells by using the Student's $t$-test ( ${ }^{\star} P<0.05, N=3$ ). (e) RT-PCR analysis of Bax, Cd95, TAp63, Krt5, Dsc2, Tacstd2 and Gapdh. (f) A bar graph showing relative mRNA levels. Significant differences were identified between control and Tacstd2-silenced HaCaT cells, between Tacstd2-silenced HaCaT cells and TAp63 rescued cells by using the Student's $t$-test $\left({ }^{*} P<0.05, N=3\right)$

associated with SCC progression. This stepwise progression of SCC could be observed clearly in pathological samples collected from the cervix. ${ }^{27,28}$ In our studies, a gradual decrease in the TACSTD2-positive cell population in the upper layers of squamous epithelia was found during the early stages of cervical SCC progression, from CON, CIN1 to CIN2/3. In SCCs, TACSTD2 staining was heterogenous and was detected in some well-differentiated epithelial cells. Compared with poorly differentiated SCCs, a higher percentage of TACSTD2-positive cells was identified in moderately differentiated SCCs, indicating that loss of TACSTD2 is a hallmark of the stepwise progression of SCC. This was consistent with previous studies, demonstrating lower expression levels of Tacstd2 in highly malignant cells, compared with immortalized keratinocytes. ${ }^{29}$ Our data demonstrated that TACSTD2 could be potentially used as a marker for more accurate and sensitive pathological grading of SCC.
On a smaller set of samples, loss of TACSTD2 was identified in poorly differentiated SCCs of head-and-neck and esophageal tumors, indicating that the loss of TACSTD2 could be a common molecular event associated with SCC. Population-based research studies will need to be initiated to verify this observation.

TACSTD2 is not required for keratinocyte differentiation. Disruption of keratinocyte differentiation has critical roles in SCC progression. Although our data demonstrated that expression of Tacstd2 was identified in differentiated keratinocyte, inhibition of Tacstd2 did not affected expression of genes associated with keratinocyte differentiation, including $\mathrm{Krt10}$, Sfn and $\mathrm{IVI}$, indicating TACSTD2 is not a key regulator of keratinocyte differentiation. These findings were consistent with the published report that TACSTD2 null mice display no overt abnormalities. ${ }^{6}$ Although expression levels of Krt5 and Dsc2, markers of the undifferentiated 
keratinocytes, were identified to be significantly induced upon Tacstd2 inhibition, indicating that lack of Tacstd2 expression could facilitate SCC progression, at least in part, by maintaining keratinocyte in an undifferentiated status. Consistent with this, our studies demonstrated that Tacstd2 expression is not presented in the undifferentiated basal cells.

Loss of TACSTD2 promotes SCC progression through attenuating TAp63-mediated apoptosis. Disruption of apoptosis has a profound effect on SCC progression and treatment resistance. ${ }^{30}$ Our data demonstrated that inhibition of Tacstd2 decreased protein level of cleaved caspase-3 and expression of pro-apoptotic genes, including TAp63, Bax and Cd95. Furthermore, the decreased activation of caspase-3, as well as Bax and Cd95 expression by Tacstd2 inhibition was rescued by induction of TAp63 expression, indicating TACSTD2 regulated keratinocyte apoptosis through TAp63 function. This functional link between TACSTD2 and TAp63 was supported by their phenotypic similarities as demonstrated in our study as well as in previous studies: neither TACSTD2 nor TAp63 was required for development of stratified squamous epithelia; expression of both could be induced by calcium signaling; and each was required for chemotherapeutic reagent-induced apoptosis. ${ }^{18}$ Our studies demonstrated that inhibition of Tacstd2 decreased gemcitabine-induced apoptosis significantly, indicating that TACSTD2 has an indispensible role in chemo-resistance of SCC.

Conclusion. SCC is a complex ecosystem composing biologically different epithelial cell populations that result in different outcomes for SCC. In-depth pathophysiological evaluation of SCC progression and exploration of the associated molecular mechanism are critical for early diagnosis and personalized treatment of SCC. Our current findings demonstrated that gradual loss of TACSTD2 is a hallmark of SCC progression, loss of TACSTD2 inhibited chemotherapeutic reagent-induced keratinocyte apoptosis. These data provide important insights that will be useful in the diagnosis and treatment of SCC.

\section{Materials and Methods}

Clinical samples. Ethical approval was obtained from the research ethics committees in Sichuan and Suzhou. Pathology samples were collected from patients who did not undergo any chemo- or radio-therapeutic treatment before biopsies or surgeries. Cervical samples $(n=56)$ were obtained at West China Second University Hospital of Sichuan University. Poorly differentiated SCC samples of esophagus $(n=5)$ and head-and-neck tumors $(n=6)$ were obtained at the First Affiliated Hospital of Soochow University and West China University Hospital. Written informed consent was obtained from all the patients. Tissue sections were prepared as previously described. ${ }^{31}$ Hematoxylin and eosin-stained slides were reviewed, and the diagnoses were confirmed independently by two pathologists according to World Health Organization standard. Two sections per sample were used for immunohistochemistry analysis of TACSTD2.

Immunohistochemistry and immunofluorescence. Tissue sections and cells cultured on coverslips were immunostained as previously described. ${ }^{32-34}$ Primary antibodies are listed in Supplementary Table 1. Biotinylated (Vector Laboratories, Burlingame, CA, USA) and fluorochrome-conjugated secondary antibodies (Alexa Fluor 488 or 594, Invitrogen, Grand Island, NY, USA) were used.

Cell culture and transfection. HaCaT cells (CLS, Eppelheim, Germany) and SiHa cells (ATCC, Manassas, VA, USA) were cultured in DMEM (Invitrogen), supplemented with L-glutamine, 10\% fetal bovine serum and penicillin/streptomycin.
Tacstd2 siRNA (Invitrogen: oligo ID HSS106222 or oligo ID HSS106223), nontargeting siRNA control (Invitrogen: cat.\# 12935-300) and transfection control siRNAs (Invitrogen: cat.\# 2013), PCDNA3.1 (+) and pcDNA3.1 ( + )-TA p63 were prepared. Transfections were performed using Lipofectamine 2000 (Invitrogen).

Protein and RNA quantification. Total proteins were prepared and western blot was performed. Antibodies used are listed in Supplementary Table S1. RNAs were isolated by using TRIZOL2000 (Invitrogen), and reverse transcribed by using VersoTM cDNA kit (Thermo Fisher Scientific, Waltham, MA, USA). The cDNAs were quantified by RT-PCR. Primers used are listed in Supplementary Table 2. Independently repeated experiments were performed $(N=3)$, and data were expressed as the mean \pm S.E.M.

Cell apoptosis assay. Cells were seeded into plates, and $48 \mathrm{~h}$ after transfection, gemcitabine hydrochloride (Eli Lilly, Indianapolis, IN, USA) was added to the culture medium (final concentration as $100 \mathrm{nM} / \mathrm{ml}$ ) to induce apoptosis for $24 \mathrm{~h}$. Cells were harvested and stained with Annexin V-FITC and propidium iodide (Calbiochem, San Diego, CA, USA), and then analyzed on BD FACS Calibur flow cytometer (BD Biosciences, San Jose, CA, USA). The percentage of apoptotic cells was calculated as apoptotic cell number $(\mathrm{Q} 2+\mathrm{Q} 4)$ /total cell number for each sample. Independently repeated experiments were performed $(N=3)$ and data were expressed as the mean \pm S.E.M.

Quantification of TACSTD2 staining. Cervical SCCs were divided into clinically relevant groupings according to ASCCP consensus guidelines, including CON (normal), CIN1 (low-grade intraepithelial lesions), CIN2/3 (high-grade cervical cancer precursor lesions) and SCC ${ }^{27}$ Cervical tissues used included 9 samples of CIN1, 27 samples of CIN2/3 and 20 samples of SCCs (5 moderately differentiated SCCs and 15 poorly differentiated SCCs). Twenty-three morphologically normal samples of stratified epithelium adjacent to the cervical lesions were used as controls.

Representative pictures were taken of the TACSTD2 stained sections: one picture per CON and CIN sample; five pictures randomly acquired per SCC sample. The area covered by epithelial cells with membrane staining for TACSTD2 (PA) and the total epithelial area (TA) were measured as indicated in Supplementary Figure 1. The TACSTD2-negative cell ratio was calculated for each measurement, which equals to (1-PA)/TA. The average percentage for CON, CIN1, CIN2/3, SCCs, moderately differentiated SCCs and poorly differentiated SCCs were calculated and expressed as the mean \pm S.E.M.

Statistical analysis. All data were analyzed using SAS software (version 9.0; SAS Institute, Cary, NC, USA). A $P$-value $\leq 0.05$ was considered significant.

\section{Conflict of Interest}

The authors declare no conflict of interest.

Acknowledgements. This research was supported by Program for New Century Excellent Talents in University (NCET-10-0572, HW), National Natural Science Foundation of China (81270057, HW) and Fundamental Research Funds for the Central University (2010SCU23009, HW). We gratefully acknowledge the support from the Program for Changjiang Scholars and Innovative Research Team in University (PCSIRT, no. 0935), from State Key Laboratory of Molecular Oncology, Chinese Academy of Medical Sciences. We thank Xueguang Zhang for critical review of the manuscript and Susan Wert for editorial help.

\section{Author contributions}

$\mathrm{H}$ Wan, $\mathrm{P}$ Yang and $\mathrm{H} \mathrm{Li}$ initiated and designed the project; $\mathrm{H}$ Wan, $\mathrm{F}$ Wang, $\mathrm{X}$ Liu and $C$ Liu performed the research; $L$ Guo, $Y$ Sheng, $P$ Yang, $H$ Li, S Long and X Liu performed the clinic-related work and pathological analysis; $\mathrm{H}$ Wan, $\mathrm{F}$ Wang and $\mathrm{X}$ Liu wrote the paper. All authors critically reviewed and approved the manuscript before submission.

1. Agada FO, Patmore H, Alhamarneh O, Stafford ND, Greenman J. Genetic profile of head and neck squamous cell carcinoma: clinical implications. J Laryngol Otol 2009; 123: 266-272.

2. Marinkovich MP. Tumour microenvironment: laminin 332 in squamous-cell carcinoma. Nat Rev Cancer 2007; 7: 370-380. 
3. Yan W, Wistuba II, Emmert-Buck MR, Erickson HS. Squamous cell carcinoma - similarities and differences among anatomical sites. Am J Cancer Res 2011; 1: 275-300.

4. Stepan LP, Trueblood ES, Hale K, Babcook J, Borges L, Sutherland CL. Expression of Trop2 cell surface glycoprotein in normal and tumor tissues: potential implications as a cancer therapeutic target. J Histochem Cytochem 2011; 59: 701-710.

5. Nakatsukasa M, Kawasaki S, Yamasaki K, Fukuoka H, Matsuda A, Tsujikawa M et al. Tumor-associated calcium signal transducer 2 is required for the proper subcellular localization of claudin 1 and 7 : implications in the pathogenesis of gelatinous drop-like corneal dystrophy. Am J Pathol 2010; 177: 1344-1355.

6. Wang J, Zhang K, Grabowska D, Li A, Dong Y, Day R et al. Loss of Trop2 promotes carcinogenesis and features of epithelial to mesenchymal transition in squamous cell carcinoma. Mol Cancer Res 2011; 9: 1686-1695.

7. Wang J, Day R, Dong Y, Weintraub SJ, Michel L. Identification of Trop-2 as an oncogene and an attractive therapeutic target in colon cancers. Mol Cancer Ther 2008; 7: 280-285.

8. Ripani E, Sacchetti A, Corda D, Alberti S. Human Trop-2 is a tumor-associated calcium signal transducer. Int J Cancer 1998; 76: 671-676.

9. McNairn AJ, Guasch G. Epithelial transition zones: merging microenvironments, niches, and cellular transformation. Eur J Dermatol 2011; 21(Suppl 2): 21-28.

10. Micallef $L$, Belaubre F, Pinon A, Jayat-Vignoles C, Delage $C$, Charveron $M$ et al. Effects of extracellular calcium on the growth-differentiation switch in immortalized keratinocyte $\mathrm{HaCaT}$ cells compared with normal human keratinocytes. Exp Dermatol 2009; 18: 143-151.

11. Schoop VM, Mirancea N, Fusenig NE. Epidermal organization and differentiation of $\mathrm{HaCaT}$ keratinocytes in organotypic coculture with human dermal fibroblasts. $J$ Invest Dermatol 1999; 112: 343-353.

12. Cheng J, Syder AJ, Yu QC, Letai A, Paller AS, Fuchs E. The genetic basis of epidermolytic hyperkeratosis: a disorder of differentiation-specific epidermal keratin genes. Cell 1992; 70 : 811-819.

13. Simpson CL, Patel DM, Green KJ. Deconstructing the skin: cytoarchitectural determinants of epidermal morphogenesis. Nat Rev Mol Cell Biol 2011; 12: 565-580.

14. Herron BJ, Liddell RA, Parker A, Grant S, Kinne J, Fisher JK et al. A mutation in stratifin is responsible for the repeated epilation (Er) phenotype in mice. Nat Genet 2005; 37: 1210-1212.

15. Byrne C, Tainsky M, Fuchs E. Programming gene expression in developing epidermis. Development 1994; 120: 2369-2383.

16. Koster MI, Kim S, Mills AA, DeMayo FJ, Roop DR. p63 is the molecular switch for initiation of an epithelial stratification program. Genes Dev 2004; 18: 126-131.

17. Koster MI, Roop DR. Mechanisms regulating epithelial stratification. Annu Rev Cell Dev Biol 2007; 23: 93-113.

18. Gressner O, Schilling T, Lorenz K, Schulze Schleithoff E, Koch A, Schulze-Bergkamen $\mathrm{H}$ et al. TAp63alpha induces apoptosis by activating signaling via death receptors and mitochondria. EMBO J 2005; 24: 2458-2471.

19. Udayanga KG, Miyata H, Yokoo Y, Qi WM, Takahara E, Mantani Y et al. Immunohistochemical study of the apoptosis process in epidermal epithelial cells of rats under a physiological condition. Histol Histopathol 2011; 26: 811-820.
20. Peter ME, Budd RC, Desbarats J, Hedrick SM, Hueber AO, Newell MK et al. The CD95 receptor: apoptosis revisited. Cell 2007; 129: 447-450.

21. Oltvai ZN, Milliman $\mathrm{CL}$, Korsmeyer SJ. Bcl-2 heterodimerizes in vivo with a conserved homolog, Bax, that accelerates programmed cell death. Cell 1993; 74: 609-619.

22. Scholzen T, Gerdes J. The Ki-67 protein: from the known and the unknown. J Cell Physiol 2000; 182: 311-322

23. Lin J, Jinno S, Okayama H. Cdk6-cyclin D3 complex evades inhibition by inhibitor proteins and uniquely controls cell's proliferation competence. Oncogene 2001; 20: 2000-2009.

24. Bold RJ, Chandra J, McConkey DJ. Gemcitabine-induced programmed cell death (apoptosis) of human pancreatic carcinoma is determined by Bcl-2 content. Ann Surg Oncol 1999; 6: 279-285.

25. Benasso M, Corvo R, Ponzanelli A, Sanguineti G, Ricci I, Pallestrini E et al. Alternating gemcitabine and cisplatin with gemcitabine and radiation in stage IV squamous cell carcinoma of the head and neck. Ann Oncol 2004; 15: 646-652.

26. Suh EK, Yang A, Kettenbach A, Bamberger C, Michaelis AH, Zhu Z et al. p63 protects the female germ line during meiotic arrest. Nature 2006; 444: 624-628.

27. Wright Jr TC, Cox JT, Massad LS, Carlson J, Twiggs LB, Wilkinson EJ. 2001 Consensus guidelines for the management of women with cervical intraepithelial neoplasia. J Low Genit Tract Dis 2003; 7: 154-167.

28. Horn LC, Klostermann K. [Precancerous lesions of the uterine cervix: morphology and molecular pathology]. Pathologe 2011; 32(Suppl 2): 242-254; Prakanzerosen der Cervix uteri: Morphologie und Molekularpathologie.

29. El Sewedy T, Fornaro M, Alberti S. Cloning of the murine TROP2 gene: conservation of a PIP2-binding sequence in the cytoplasmic domain of TROP-2. Int J Cancer 1998; 75: 324-330.

30. Lowe SW, Lin AW. Apoptosis in cancer. Carcinogenesis 2000; 21: 485-495.

31. Wan H, Xu Y, Ikegami M, Stahlman MT, Kaestner KH, Ang SL et al. Foxa2 is required for transition to air breathing at birth. Proc Natl Acad Sci USA 2004; 101: 14449-14454.

32. Dave V, Wert SE, Tanner T, Thitoff AR, Loudy DE, Whitsett JA. Conditional deletion of Pten causes bronchiolar hyperplasia. Am J Respir Cell Mol Biol 2008; 38: 337-345.

33. Tompkins DH, Besnard V, Lange AW, Keiser AR, Wert SE, Bruno MD et al. Sox2 activates cell proliferation and differentiation in the respiratory epithelium. Am J Respir Cell Mol Biol 2011; 45: 101-110.

34. Wan H, Luo F, Wert SE, Zhang L, Xu Y, Ikegami M et al. Kruppel-like factor 5 is required for perinatal lung morphogenesis and function. Development 2008; 135: 2563-2572.

(c) (i) Cell Death and Disease is an open-access journal published by Nature Publishing Group. This work is licensed under a Creative Commons Attribution-NonCommercialShareAlike 3.0 Unported License. To view a copy of this license, visit http://creativecommons.org/licenses/by-nc-sa/3.0/

Supplementary Information accompanies this paper on Cell Death and Disease website (http://www.nature.com/cddis) 